NUB-3154/97-Th

Jan 1997

hep-ph/9701373

\title{
From Planck to GUT via Dimensional Transmutation
}

\author{
Haim Goldberg \\ Department of Physics \\ Northeastern University \\ Boston, MA 02115
}

\begin{abstract}
Consider a gauge singlet superfield $S$ coupled to a pair of adjoint fields in a SUSY-GUT. If the tree-level vacuum is flat in $S$, the vev $\langle S\rangle$ which defines the GUT scale will be determined via dimensional transmutation at a scale $M$ where the soft-breaking (mass) ${ }^{2}$ vanishes as a result of running from $M_{P}=\left(8 \pi G_{N}\right)^{-1 / 2}$. Because of the large number of adjoint fields $\mathcal{N}_{A}$ coupled to $S$, one finds that $M$ can be generically close to $M_{\mathrm{GUT}}=2 \times 10^{16} \mathrm{GeV}$ :$$
M \simeq M_{P} \exp \left[-16 \pi^{2} \log (3 / 2) /\left(\mathcal{N}_{A}+4\right) \lambda^{2}\right]
$$

where $\lambda$ is a Yukawa $\sim 0.7$. This work examines the symmetries and dynamical constraints required in a SUSY-GUT in order that the desired flatness in $S$ is achieved, and that this flatness may survive in a supergravity framework.
\end{abstract}




\section{Introduction}

The realization of a cogent supersymmetric grand unified theory (SUSY-GUT) has constituted an important goal in particle theory for well over a decade. Several impediments to achieving such a goal were already evident in the earliest papers on the subject [1], 2]. In $\mathrm{SU}(5)$, these consist of the lack of a mechanism within the theory to $(a)$ lift the degeneracy of (supersymmetric) SU(5)-, $\mathrm{SU}(4) \times \mathrm{U}(1)$-, and $\mathrm{SU}(3) \times \mathrm{SU}(2) \times \mathrm{U}(1)$ invariant ground states and $(b)$ implement a hierarchical splitting of the massless Higgs doublets from the massive triplets ( $\mathrm{D} / \mathrm{T}$ splitting) in the $\mathbf{5}+\overline{\mathbf{5}}$ representations. The lifting of the degeneracy is generally ascribed to the perturbation of the various ground states by the soft-breaking terms [1, 3], while the doublet-triplet splitting can be effected through a judicious choice of the Higgs sector [4].

When the SUSY-GUT is considered in the context of string theory, an additional problem emerges - the origin of the GUT scale as distinct from the Planck or string scale [5]. Generally speaking, the construction of the superpotential in string theory does not in any obvious way allow for the introduction of an additional scale $M$, as is manifest in the $\mathrm{SU}(5)$ superpotential

$$
W=M \operatorname{Tr} A^{2}+\lambda \operatorname{Tr} A^{3},
$$

where $A$ is the $\mathrm{SU}(5)$ adjoint chiral superfield. It is $M$ that sets the scale $M_{\mathrm{GUT}} \simeq$ $10^{16}-10^{17} \mathrm{GeV}$ of $\mathrm{SU}(5)$-breaking, and there is no obvious relation between $M_{\mathrm{GUT}}$ and the Planck scale $M_{P}=\left(8 \pi G_{N}\right)^{-1 / 2}=2.44 \times 10^{18} \mathrm{GeV}$ [5].

An obvious possibility is to consider, instead of (1)

$$
W^{\prime}=\lambda S \operatorname{Tr} A^{2}+\lambda^{\prime} \operatorname{Tr} A^{3}+w(S)
$$

where $S$ is an SU(5) singlet chiral superfield, and search for a mechanism which yields $\langle S\rangle \neq 0$, and $M=\lambda\langle S\rangle \sim M_{\mathrm{GUT}}$. It is clear that, without additional input, (2) as it stands is not viable, since any $Z_{N}$ (or $\mathrm{U}(1)$ ) invariance invoked in order to restrict $w(S)$ will allow $w(S) \sim S^{3}$, which, for arbitrary Yukawas, forces $\langle S\rangle=0$ at tree level .

In this work, I will examine the possibility of generating $M_{\mathrm{GUT}}$ from $M_{P}$ through radiative corrections in the soft-breaking sector, with a resulting dimensional trans- 
mutation [6] at the scale $M \simeq M_{\mathrm{GUT}}$. This will turn out to be possible, perhaps even inevitable, under certain simple, well-defined constraints placed on the superpotential. These constraints serve to effect the necessary flatness of the effective potential near the origin of $S$, so that the minimum is free to wander off to the point of dimensional transmutation, $\langle S\rangle \sim M_{\mathrm{GUT}}$.

\section{Toy Model}

To illustrate some of the salient points, I will begin with the case of only two visible sector superfields, the singlet $S$ and an adjoint $A$. For the moment, I will impose a continuous $R$-symmetry where all superfields have $R$-character $\frac{1}{3}$, so that all terms in the superpotential $W$ are trilinear. In the case of where the $\mathrm{GUT}$ is $\mathrm{SU}(N)$ the most general superpotential consistent with these requirements is (omitting couplings)

$$
W=S \operatorname{Tr} A^{2}+\operatorname{Tr} A^{3}+S^{3}
$$

In the absence of soft-breaking, the vacuum is given by $\langle S\rangle=\langle A\rangle=0$. With softbreaking, even at a point where the soft-breaking $m_{S}^{2}=0$, the vevs will be shifted to $\langle S\rangle \sim\langle A\rangle \sim m_{3 / 2}$. This could be avoided if the $S^{3}$ term were absent. However, as remarked in the introduction, any symmetry prohibiting the $S^{3}$ term will also forbid the $A^{3}$ term. Let us accept this for now, so that one can impose a $Z_{4}$ symmetry with charges $q_{4}(S)=2, q_{4}(A)=1$. As a consequence of this and the $R$-symmetry, the superpotential is determined uniquely:

$$
W_{0}=\lambda S \operatorname{Tr} A^{2}=\frac{1}{2} \lambda S \sum_{a=1}^{\mathcal{N}_{A}} A_{a} A_{a}
$$

where $\mathcal{N}_{A}=N^{2}-1$ is the dimension of the adjoint of $\mathrm{SU}(N)$. In this toy model, it is easy also to include $\mathrm{SO}(10)$ in the discussion, in which case $\mathcal{N}_{A}=45$.

At tree level, in the absence of soft-breaking, the vacuum corresponding to (4) is $\langle A\rangle=0$ with $\langle S\rangle$ undetermined. I now introduce the soft-breaking potential

$$
V_{\text {soft }}=m_{S}^{2} S^{*} S+m_{A}^{2} \sum_{a=1}^{\mathcal{N}_{A}} A_{a}^{*} A_{a}+\frac{1}{2} \lambda A S \sum_{a=1}^{\mathcal{N}_{A}} A_{a} A_{a}+\text { h.c. }+\frac{1}{2} \widetilde{M} \sum_{a=1}^{\mathcal{N}_{A}} \lambda_{a}^{T} \lambda_{a}
$$


where $\lambda_{a}$ is the adjoint gaugino, and the standard trilinear coupling parameter $A$ is (hopefully) not to be confused with the adjoint field $A_{a}$. This work will focus on the case where the RG evolution of $m_{S}^{2}$ down from $M_{P}$ leads to its vanishing at some scale $Q=M$. In that case the 1-loop improvement to the effective potential at scales near $M$ leads to the replacement in $V_{\text {soft }}$ [6]

$$
m_{S}^{2} S^{*} S \longrightarrow m^{\prime 2} S^{*} S \ln \left(S^{*} S / M^{2}\right)
$$

where $m^{\prime 2}=-\left.\frac{1}{2} \frac{d m_{S}^{2}}{d t}\right|_{M}, t \equiv \ln \left(M_{P} / Q\right)$. The potential to be minimized is

$$
V=\left|\frac{\partial W}{\partial S}\right|^{2}+\left|\frac{\partial W}{\partial A_{a}}\right|^{2}+\frac{1}{2} m^{\prime 2} S^{*} S \ln \left(S^{*} S / M^{2}\right)+m_{A}^{2} A_{a}^{*} A_{a}+\frac{1}{2} \lambda A S A_{a} A_{a}+\text { h.c. }
$$

where in accordance with $D$-flatness the adjoint field is chosen along one of the directions of the Cartan subalgebra. There is no sum on $a$ in Eq. (7).

It is a matter of algebra to see that even in the presence of the soft breaking, $V$ is minimized for $\left\langle A_{a}\right\rangle=0$. However, $\langle S\rangle$ is now determined: one obtains $\langle S\rangle=M / \sqrt{\mathrm{e}}$, so that although the gauge symmetry remains unbroken, the adjoint field grows a mass $\lambda M / \sqrt{\mathrm{e}}$. This is dimensional transmutation, the breaking of scale invariance due to renormalization effects. It will now be seen that for generic choices of parameters, the RG equations will drive $m_{S}^{2}$ negative at a scale $M \sim M_{\mathrm{GUT}}$.

The RG equations for this model are straightforward to obtain:

$$
\begin{aligned}
16 \pi^{2} \frac{d g}{d t} & =-\left(\sum S_{2}(R)-3 C_{2}(a d j)\right) g^{3} \\
16 \pi^{2} \frac{d \widetilde{M}}{d t} & =-2\left(\sum S_{2}(R)-3 C_{2}(a d j)\right) g^{2} \widetilde{M} \\
16 \pi^{2} \frac{d \lambda}{d t} & =-\frac{1}{2} \lambda\left[\left(\mathcal{N}_{A}+4\right) \lambda^{2}-8 g^{2} C_{2}(a d j)\right] \\
16 \pi^{2} \frac{d A}{d t} & =-\lambda\left[\left(\mathcal{N}_{A}+4\right) \lambda^{2} A+8 g^{2} C_{2}(\operatorname{adj}) \widetilde{M}\right] \\
16 \pi^{2} \frac{d m_{S}^{2}}{d t} & =-\mathcal{N}_{A} \lambda^{2}\left(m_{S}^{2}+2 m_{A}^{2}+A^{2}\right) \\
16 \pi^{2} \frac{d m_{A}^{2}}{d t} & =-2 \lambda^{2}\left(m_{S}^{2}+2 m_{A}^{2}+A^{2}\right)+8 g^{2} C_{2}(\operatorname{adj}) \widetilde{M}^{2},
\end{aligned}
$$


where $C_{2}(a d j)=N$ for $\mathrm{SU}(N), 8$ for $\mathrm{SO}(10)$, and $S_{2}(R)$ is the Dynkin index of any gauge-coupled field. As defined previously, $\mathcal{N}_{A}$ is the dimension of the adjoint. Standard initial conditions are imposed on the soft scalar masses: $m_{S}^{2}\left(M_{P}\right)=m_{A}^{2}\left(M_{P}\right)=$ $m_{0}^{2}$. For simplicity of discussion, I will assume in all that follows that the quantity $\sum S_{2}(R)$ is such that the gauge coupling is essentially constant between $M_{P}$ and the scale $M$. As a result, the gaugino mass $\widetilde{M}$ will also be constant.

Examination of the evolution equation for $m_{S}^{2}$ in (8) reveals immediately why dimensional transmutation is likely to occur at scale $M$ not far below $M_{P}$ :

- there is a large factor of $\mathcal{N}_{A}$ multiplying the right hand side:

- there is no gaugino contribution serving to retard the decrease of $m_{S}^{2}$ with momentum scale.

Neither of these properties characterize the $m_{A}^{2}$ equation, and both are directly tied to the gauge singlet nature of $S$. A simple analytic treatment is heuristic: take $A=$ $\widetilde{M}=0, \lambda=$ constant. (The latter will be strictly true only at the fixed point.) Then a simple integration of the last two of Eqs. (8) gives the solution

$$
\frac{m_{S}^{2}}{m_{0}^{2}}=1-3\left(\frac{\mathcal{N}_{A}}{\mathcal{N}_{A}+4}\right)\left(1-e^{-\kappa t}\right)
$$

where $\kappa=\left(\left(\mathcal{N}_{A}+4\right) \lambda^{2} / 16 \pi^{2}\right)$. Thus, to a good approximation, $m_{S}^{2}=0$ at $t_{1}=$ $\ln (3 / 2) / \kappa$, or

$$
Q_{1}=M=M_{P} e^{-16 \pi^{2} \log (3 / 2) /\left(\mathcal{N}_{A}+4\right) \lambda^{2}} .
$$

Because of the large size of $\mathcal{N}_{A}+4$, the evolution to the point of dimensional transmutation is rapid: from Eq. (10), $M=M_{\mathrm{GUT}}$ in $\mathrm{SU}(5)$ for $\lambda \simeq 0.7$.

For $A, \widetilde{M} \neq 0$, some representative numbers can be given. With $g^{2} / 4 \pi=1 / 24$, $A\left(M_{P}\right)=\widetilde{M}\left(M_{P}\right)=m_{0}$, I find $m_{S}^{2}=0$ at $M=M_{\mathrm{GUT}}$ for $\lambda\left(M_{P}\right)=0.57$ in the case of $\mathrm{SU}(5)$, and $\lambda\left(M_{P}\right)=0.34$ in the case of $\mathrm{SO}(10)$. There are sizeable arrays of parameter space for which $M \simeq M_{\mathrm{GUT}}$, and I will present more detail in the discussion of a more realistic model. Two points may be noted before proceeding: 
- The GUT scale is triggered by dimensional transmutation in the soft-breaking sector, but it has no explicit or implicit dependence on $m_{3 / 2}$ : it is essentially given by Eq. (10).

- Renormalizability is crucial to the dynamical mechanism proposed here. Thus it is unclear how to relax the requirement of continuous $R$-invariance so as to allow higher dimension operators (such as $\left.\left(S \operatorname{Tr} A^{2}\right)^{n} / M_{P}^{3(n-1)}\right)$ which respect the $Z_{4}$ or U(1) symmetry requirement. Such terms may also vitiate $F$-flatness in $S$. For this paper, I maintain the strict $R$-invariance of the superpotential.

\section{Model with Gauge Symmetry Breaking}

A more realistic model requires some mechanism for the breaking of $\mathrm{SU}(N)$. (The $\mathrm{SO}(10)$ case will receive comment later.) As already noted, simply extending the original SU(5) model by letting $M \rightarrow S$ is not possible, since undesirable $S^{3}$ terms are then permitted in the superpotential. Instead, it is necessary to introduce a second adjoint $A^{\prime}$, and take as the superpotential

$$
W=2 \lambda S \operatorname{Tr} A A^{\prime}+2 \lambda^{\prime} \operatorname{Tr} A^{2} A^{\prime},
$$

with the $Z_{4}$ assignments $\left(S, A, A^{\prime}\right)=(1,1,2)$. Once more, a continuous $R$-symmetry is

imposed, with $R$-character $=\frac{1}{3}$ for all fields, which will forbid terms such as $M \operatorname{Tr} A^{\prime 2}$, as well as all higher dimensional operators consistent with the $Z_{4}$ symmetry.

At tree level, the vacuum configuration for $\mathrm{SU}(5)$ in the direction of the standard model (the "24" direction) is given by

$$
\langle A\rangle=\left(\lambda / \lambda^{\prime}\right)\langle S\rangle \operatorname{diag}(2,2,2,-3,-3), \quad\left\langle A^{\prime}\right\rangle=0,
$$

with $\langle S\rangle$ undetermined.

The soft-breaking potential is now generalized to

$$
\begin{aligned}
V_{\text {soft }}= & m_{S}^{2} S^{*} S+\sum_{a=1}^{\mathcal{N}_{A}}\left(m_{A}^{2} A_{a}^{*} A_{a}+m_{A^{\prime}}^{2} A_{a}^{\prime *} A_{a}^{\prime}+\lambda A S A_{a} A_{a}^{\prime}+\text { h.c. }\right) \\
& +\frac{1}{2} A^{\prime} \lambda^{\prime} \sum_{a, b, c}^{\mathcal{N}_{A}} d^{a b c} A_{a} A_{b} A_{c}^{\prime}+\text { h.c. }+\frac{1}{2} \sum_{a=1}^{\mathcal{N}_{A}} \widetilde{M} \lambda_{a}^{T} \lambda_{a},
\end{aligned}
$$


where the $d^{a b c}$ is the symmetric $\mathrm{SU}(N)$ tensor. The RG equations for this model are:

$$
\begin{aligned}
16 \pi^{2} \frac{d \lambda}{d t}= & \frac{1}{2} \lambda\left[\left(N^{2}+3\right) \lambda^{2}+3 N^{\prime} \lambda^{\prime 2}-8 g^{2} N\right] \\
16 \pi^{2} \frac{d \lambda^{\prime}}{d t}= & \frac{1}{2} \lambda^{\prime}\left[6 \lambda^{2}+5 N^{\prime} \lambda^{\prime 2}-12 g^{2} N\right] \\
16 \pi^{2} \frac{d A}{d t}= & 2\left[\left(N^{2}+1\right) \lambda^{2} A+N^{\prime} \lambda^{\prime 2} A^{\prime}+4 g^{2} N \widetilde{M}\right] \\
16 \pi^{2} \frac{d A^{\prime}}{d t}= & 2\left[\lambda^{2} A+\frac{3}{2} N^{\prime} \lambda^{\prime 2} A^{\prime}+6 g^{2} N \widetilde{M}\right] \\
16 \pi^{2} \frac{d m_{S}^{2}}{d t}= & \left(N^{2}-1\right) \lambda^{2}\left[m_{S}^{2}+2\left(m_{A}^{2}+m_{A^{\prime}}^{2}+A^{\prime 2}\right)\right] \\
16 \pi^{2} \frac{d m_{A}^{2}}{d t}= & 2\left[\left(\lambda^{2} m_{S}^{2}+\left(\lambda^{2}+2 N^{\prime} \lambda^{\prime 2}\right) m_{A}^{2}+\left(\lambda^{2}+N^{\prime} \lambda^{\prime 2}\right) m_{A^{\prime}}^{2}\right.\right. \\
& \left.+\lambda^{2} A^{2}+N^{\prime} \lambda^{\prime 2} A^{\prime 2}-4 g^{2} N \widetilde{M}^{2}\right] \\
16 \pi^{2} \frac{d m_{A^{\prime}}^{2}}{d t}= & 2\left[\left(\lambda^{2} m_{S}^{2}+\left(\lambda^{2}+N^{\prime} \lambda^{\prime 2}\right) m_{A}^{2}+\left(\lambda^{2}+\frac{1}{2} N^{\prime} \lambda^{\prime 2}\right) m_{A^{\prime}}^{2}\right.\right. \\
& \left.+\lambda^{2} A^{2}+\frac{1}{2} N^{\prime} \lambda^{\prime 2} A^{\prime 2}-4 g^{2} N \widetilde{M}^{2}\right],
\end{aligned}
$$

where $N^{\prime}=\left(N^{2}-4\right) / N$. Once again, one notes the large $\mathcal{N}_{A}=N^{2}-1$ factor, as well as the absence of the gaugino mass term on the R.H.S. of the $m_{S}^{2}$ equation in (14), allowing, as in the toy model, a rapid evolution of $m_{S}^{2}$ toward zero. In the present case, there are factors of $O(N)$ enhancing the decrease of $m_{A}^{2}, m_{A^{\prime}}^{2}$ in descending from $M_{P}$. Nevertheless, unless $\widetilde{M}=0$ and $\lambda^{\prime}\left(M_{P}\right) \geq 1.5$, these quantities will not be driven negative in the region above $10^{16} \mathrm{GeV}$.

\section{Numerical Study}

In Figure 1, I show some sample ranges of parameters which will give dimensional transmutation at $M=M_{\mathrm{GUT}}$ in $\mathrm{SU}(5)$. The numerical data are presented as loci in the $\lambda\left(M_{P}\right)-\lambda^{\prime}\left(M_{P}\right)$ space for the four sets of initial conditions $A\left(M_{P}\right)=A^{\prime}\left(M_{P}\right)=\left(0, m_{0}\right)$, and $\widetilde{M}\left(M_{P}\right)=\left(0, m_{0}\right)$. The gauge coupling $g^{2} / 4 \pi$ is again fixed at $1 / 24$. The required values of the Yukawa $\lambda\left(M_{P}\right)$ are all in the range $0.4-0.6$, showing that the dynamics is effectively controlled by the physics already present in the toy model of the last section. 


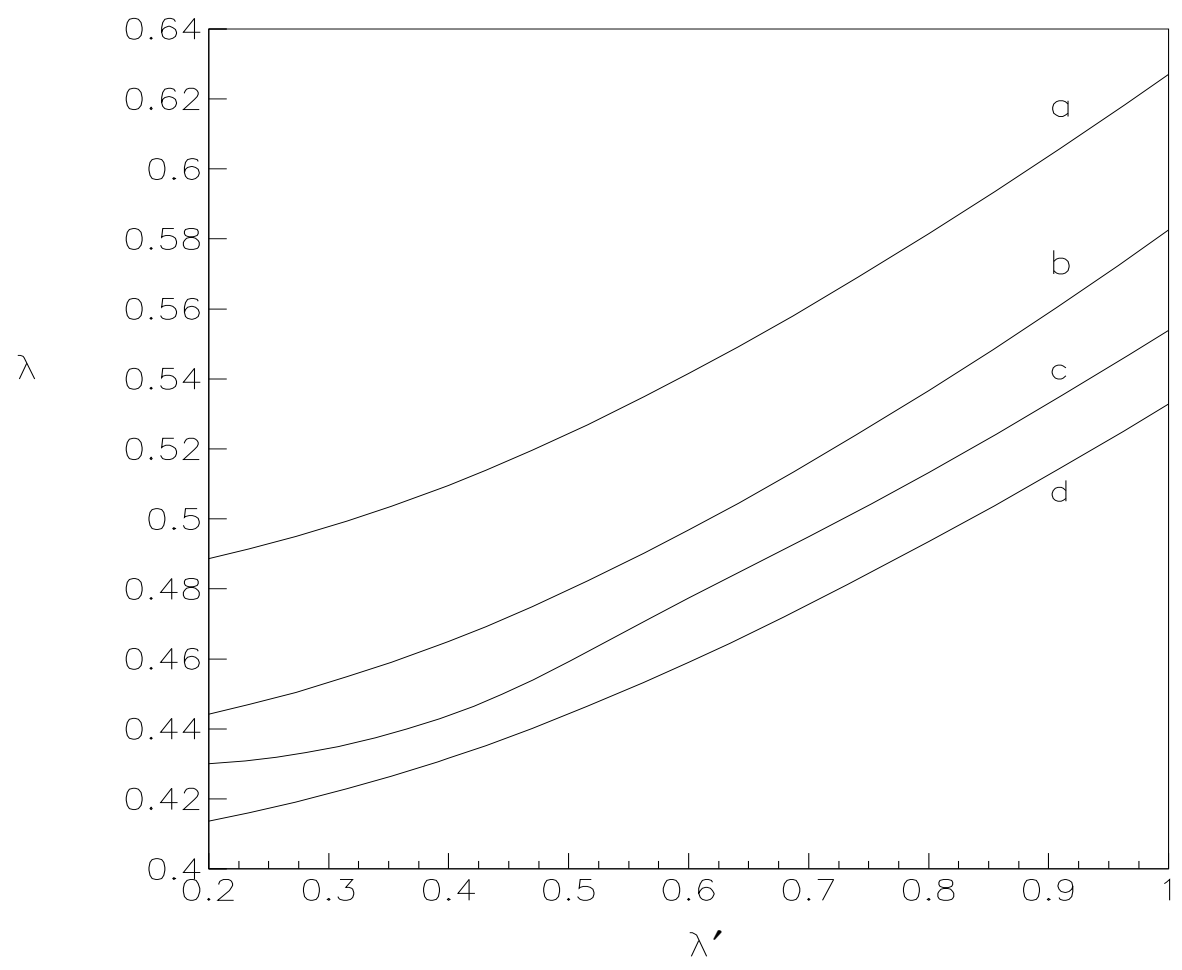

Figure 1: Loci in space of Yukawa couplings giving dimensional transmutation at $2 \times 10^{16} \mathrm{GeV}$, for various choices of gaugino mass $\widetilde{M}\left(M_{P}\right)$ and trilinear parameters $A\left(M_{P}\right), A^{\prime}\left(M_{P}\right)$ at the Planck scale. Curve $(a):\left(\widetilde{M}, A, A^{\prime}\right)=(0.5,0,0) m_{0} ;(b)$ : $\left(\widetilde{M}, A, A^{\prime}\right)=(0.5,1,1) m_{0} ;(c):\left(\widetilde{M}, A, A^{\prime}\right)=(1,0,0) m_{0} ;(d):\left(\widetilde{M}, A, A^{\prime}\right)=$ $(1,1,1) m_{0}$;

It is straightforward to check the spectrum of this model at scales $Q<M$ : There are 24 Dirac spinors and superpartners with GUT-scale masses, and one light standard model singlet chiral field. The scalar component of this field presents a potential Polonyi problem, which will receive some comment in the concluding section. 


\section{Effects of Supergravity}

To what extent are the results presented here stable with respect to extension to local supersymmetry (supergravity)? In a $D$-flat direction, the tree-level potential in local supersymmetry (for the visible sector only) corresponding to a superpotential $W\left(Z_{i}\right)$ is given by [7]

$$
V_{\text {sugra }}=e^{K / M_{P}^{2}}\left[\left(\frac{\partial W}{\partial Z_{i}}+\frac{\partial K}{\partial Z_{i}} \frac{W}{M_{P}^{2}}\right)\left(K^{-1}\right)_{i j^{*}}\left(\frac{\partial W}{\partial Z_{j}}+\frac{\partial K}{\partial Z_{j}} \frac{W}{M_{P}^{2}}\right)^{*}-3 \frac{W W^{*}}{M_{P}^{2}}\right],
$$

where $K\left(Z_{i}, Z_{i}^{*}\right)$ is the Kähler potential and $K^{-1}$ is the inverse of the matrix $K_{i j^{*}} \equiv$ $\partial^{2} K / \partial Z_{i} \partial Z_{j}^{*}$. The $R$-symmetry restriction to superpotentials of homogeneous degree 3 implies

$$
\sum_{i} Z_{i} \frac{\partial W}{\partial Z_{i}}=3 W
$$

For a flat Kähler $\left(K=\sum_{i} Z_{i} Z_{i}^{*}\right)$, one obtains on inserting (16) into (15)

$$
V_{\text {sugra }}=\exp \left(\sum_{i}\left|Z_{i}\right|^{2} / M_{P}^{2}\right)\left[\sum_{i}\left|\frac{\partial W}{\partial Z_{i}}\right|^{2}+\left(3+\sum_{i} \frac{\left|Z_{i}\right|^{2}}{M_{P}^{2}}\right) \frac{|W|^{2}}{M_{P}^{2}}\right] .
$$

From (17), we find that $V_{\text {sugra }} \geq 0$. Eqs. (17) and (16) then ensure that the global symmetry condition $\partial W / \partial Z_{i}=0$ provides a necessary and sufficient condition for the minimum $(V=0)$ to be obtained. From this, it follows that if $\langle S\rangle$ is not determined in the global theory (before soft-breaking), neither is it determined in the flat-Kähler local theory.

What about higher order terms in the Kähler potential? For an arbitrary Kähler, the $R$-symmetry (16) guarantees that $\partial V_{\text {sugra }} / \partial Z_{i}=V_{\text {sugra }}=0$ at the ( $S$-flat) field configuration corresponding to $\partial W / \partial Z_{i}=0$; it does not, of course, guarantee that this field configuration provides a global minimum for the potential. There is an interesting case where it does: consider in the Toy Model of Section 2 a region of field space where $K=\rho+\frac{1}{2} a \rho^{2} / M_{P}{ }^{2}, \rho=S^{*} S+A^{*} A$. This is the $\mathrm{U}(N)(N=2)$ symmetric form suggested by graviton loop corrections [ 8 ]. If $a \geq 0$, then one can show that the minima of the global and local theories coincide, and $\langle S\rangle$ is still undetermined. Generally speaking, if $K$ is such as to destroy $S$-flatness, the vevs will be moved to $O\left(M_{P}\right)$, the 
space will become anti-deSitter, and the entire $R$-symmetry must be dropped in order to cancel the resulting $O\left(M_{P}{ }^{4}\right)$ cosmological constant.1 For now, I will just assume that $K$ behaves in a manner such as to preserve the vacuum in the $S$-flat direction, and delay consideration of this point to future study. It must be noted, however, that even if $K$ behaves appropriately, the local theory is still not renormalizable, so that the dimensional transmutation requires ignoring the gravitational strength interactions in obtaining the running of the soft parameters.

\section{Summary and Remarks}

(1) In this work, I have demonstrated how the GUT scale $M_{\mathrm{GUT}}$ could arise through dimensional transmutation at a scale $M$ where the soft-breaking $m_{S}^{2}$ of a gauge-singlet field $S$ becomes negative and a vev $\langle S\rangle=M / \sqrt{\mathrm{e}}$ develops. The scale $M$ does not depend numerically on the SUSY-breaking scale $m_{3 / 2}$, and is of $O\left(M_{\mathrm{GUT}}\right)$ because the rate of decrease of $m_{S}^{2}$ on descending from $M_{P}$ is proportional to a large number, the dimension of the adjoint Higgs representation $A$. At the scale $M$, the adjoint develops a mass $\sim M$, and if there is self-coupling, a non-zero vev.

(2) This scenario requires $S$-flatness of the effective potential before radiative corrections. In this work, this has been implemented by two symmetries: a continuous $R$-symmetry which enforces all terms in the superpotential to be trilinear, and a discrete or continuous symmetry which forbids more than a linear dependence on $S$ for any term in the superpotential. Except for possible gravitational effects discussed above, the $R$-symmetry allows the theory to be renormalizable between $M_{P}$ and $M$, while the additional symmetry keeps $\langle S\rangle$ indeterminate at tree level, allowing dimensional transmutation to take place at the high scale $\sim M_{\mathrm{GUT}}$.

(3) An extension to $\mathrm{SO}(10)$ of the second model discussed in this paper would require a third adjoint (or a symmetric 54) in order to create a trilinear term besides $S A A^{\prime}$. Such an extension, and other non-trivial modifications (such as those required to ac-

\footnotetext{
${ }^{1}$ The hidden sector does not, of course, respect an $R$-symmetry, because of the dual requirements of breaking SUSY and maintaining a zero cosmological constant.
} 
commodate the doublet triplet splitting) are the subjects of future study. It should be noted that every field coupled to the singlet $S$ will tend to drive the transmutation scale $M$ closer to $M_{P}$. This will limit the number and dimension of such fields.

(4) Many $\mathrm{SO}(10)$ models require a set of heavy $\mathbf{1 6}+\overline{\mathbf{1 6}}$ pairs of superfields to effect the $\mathrm{SO}(10) \rightarrow \mathrm{SU}(5)$ breaking, and in order to obtain realistic low energy Yukawa matrices [9]. By allowing the singlet $S$ to couple to such pairs, the dimensional transmutation will automatically force them to grow a mass $M$.

(5) The development of a vev for $S$ will break the $Z_{4}$ (or $\mathrm{U}(1)$ ) symmetry used in order to forbid the $S^{3}$ term. In the $Z_{4}$ case, the resulting domain walls can be rendered harmless by a period of post-GUT inflation. In the U(1) case, the undesirable GUTscale axion [10 is not present if the $\mathrm{U}(1)$ is gauged. The final cosmological problem is presented by the scalar component of the field $S^{\prime}=S-\langle S\rangle$, which has a mass $\sim\left|d m_{S}^{2} / d t\right|^{1 / 2} \sim m_{3 / 2}$. I If these particles survive to the post-inflation era as a longwavelength classical field with amplitude of $S^{\prime} \sim O\left(M_{\mathrm{GUT}}\right)$, then the familiar Polonyi problem results [11]. After the onset of inflation, $S^{\prime}$ has a mass $\sim H$ [12], and is localized at $S^{\prime}=0$ [12, 13]. Whether or not it remains localized depends on its Kähler couplings to the inflaton and to the fields of the hidden sector [14]. Discussion of this awaits a fuller understanding of Planck scale physics.

\section{Acknowledgement}

I would like to thank Stuart Raby for helpful comments. This research was supported in part by Grant No PHY9411546 from the National Science Foundation.

\section{References}

[1] S. Dimopoulos and H. Georgi, Nucl. Phys. B193 (1981) 150.

[2] N. Sakai, Zeit. Phys. C11 (1981) 153.

\footnotetext{
${ }^{2}$ In the two-adjoint model of Section 3, the light scalar is a linear combination of $S$ and $A_{24}^{\prime}$.
} 
[3] N. Dragon, Zeit. Phys. C15 (1982) 169.

[4] S. Dimopoulos and F. Wilczek, Proceedings of the Erice Summer School, ed. A Zichichi (1981); H. Georgi, Phys. Lett. 108B (1982) 283; A. Masiero, D. V. Nanopoulos, K. Tamvakis, and T. Yanagida, Phys. Lett. 115B (1982) 380; B. Grinstein, Nucl. Phys. B206 (1982) 387.

[5] For a comprehensive recent survey of the problem, see Keith R. Dienes, IASSNSHEP-95-97, hep-th/9602045.

[6] S. Coleman and E. Weinberg, Phys. Rev. D7 (1973) 1883. This mechanism was discussed in the context of radiative electroweak breaking in the MSSM by J. Ellis, J. Hagelin, D. V. Nanopoulos, and K. Tamvakis, Phys. Lett. 125B (1983) 275. See also J. Ellis, A. B. Lahanas, D. V. Nanopoulos, K. Tamvakis, Phys. Lett. 134B (1983) 429; C. Kounnas, A. B. Lahanas, and D. V. Nanopoulos, Nucl. Phys. B236 (1984) 438. An attempt to generate $M_{\mathrm{GUT}}$ from $M_{P}$ (B. Gato, J. Léon, and M. Quirós, Phys. Lett. 136B (1984) 361) made use of a superpotential $W \sim \eta \operatorname{Tr} A^{2}, \eta \sim O\left(m_{3 / 2}\right)$, which introduces a small mass parameter in the superpotential. More in the spirit of the present work is a paper by P. Moxhay and K. Yamamoto, Phys. Lett. 151B (1985) 363, which proposes obtaining the Peccei-Quinn scale through dimensional transmutation. There have also been previous efforts to generate $M_{\mathrm{GUT}}$ from the weak scale (E. Witten, Phys. Lett. 105B (1981) 267) and from an intermediate SUSY-breaking scale (S. Dimopoulos and S. Raby, Nucl. Phys. B219 (1983) 479) via radiative corrections.

[7] E. Cremmer, S. Ferrara, L. Girardello, and A. van Proeyen, Phys. Lett. 116B (1982) 231; Nucl. Phys. B212 (1983) 413; A. H. Chamseddine, R. Arnowitt, and P. Nath, Phys. Rev. Lett. 49 (1982) 970; E. Witten and J. Bagger, Phys. Lett. 115B (1982) 202; J. Bagger, Nucl. Phys. B211 (1983) 302.

[8] L. J. Hall, J. Lykken, and S. Weinberg, Phys. Rev. D27 (1983) 2359. 
[9] G. Anderson, S. Dimopoulos, L. J. Hall, S. Raby, and G. Starkman, Phys. Rev. D49 (1994) 3660; K. S. Babu and S. M. Barr, Phys. Rev. Lett. 75 (1995) 2088.

[10] J. Preskill, M. B. Wise, and F. Wilczek, Phys. Lett. 120B (1983) 127; L. F. Abbott and P. Sikivie, Phys. Lett. 120B (1983) 133; M. Dine and W. Fischler, Phys. Lett. 120B (1983) 137.

[11] G. Coughlan, W. Fischler, E. Kolb, S. Raby, and G. Ross, Phys. Lett. 131B (1983) 59; J. Ellis, D. V. Nanopoulos, and M. Quirós, Phys. Lett. 174B (1986); B. de Carlos, J. A. Casas, F. Quevedo, and E. Roulet, Phys. Lett. 318B (1993) 447.

[12] M. Dine, W. Fischler, and D. Nemeschansky, Phys. Lett. 136B (1984)169.

[13] G. Dvali, hep-ph/9503259, preprint IFUP-TH 09-95,

[14] M. Dine, L. Randall, and S. Thomas, Phys. Rev. Lett. 75 (1995) 398 (hep$\mathrm{ph} / 9503303)$. 